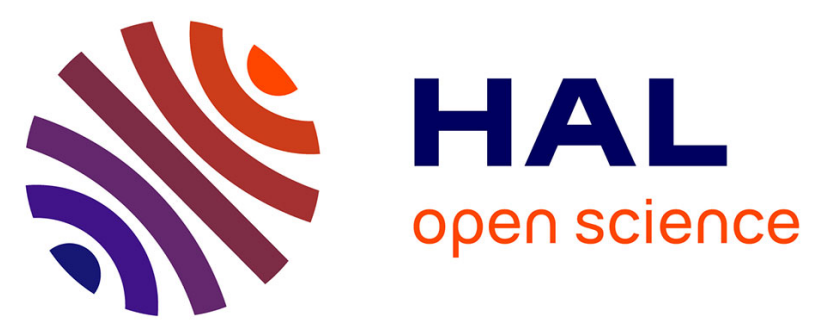

\title{
Education thérapeutique et douleur liée au cancer : stratégie et points forts du programme EFFADOL "Ensemble, Faire Face À la DOuLeur "
}

Virginie Prevost, Alexandra Leconte, Natacha Heutte, Claire Delorme, Aline Le Chevalier, Bénédicte Clarisse

\section{To cite this version:}

Virginie Prevost, Alexandra Leconte, Natacha Heutte, Claire Delorme, Aline Le Chevalier, et al.. Education thérapeutique et douleur liée au cancer : stratégie et points forts du programme EFFADOL “ Ensemble, Faire Face À la DOuLeur ”. La Presse Médicale, 2018, 47 (10), pp.921-924. 10.1016/j.lpm.2018.08.002 . inserm-02102611

\section{HAL Id: inserm-02102611 https://www.hal.inserm.fr/inserm-02102611}

Submitted on 3 Jul 2019

HAL is a multi-disciplinary open access archive for the deposit and dissemination of scientific research documents, whether they are published or not. The documents may come from teaching and research institutions in France or abroad, or from public or private research centers.
L'archive ouverte pluridisciplinaire HAL, est destinée au dépôt et à la diffusion de documents scientifiques de niveau recherche, publiés ou non, émanant des établissements d'enseignement et de recherche français ou étrangers, des laboratoires publics ou privés. 
Education thérapeutique et douleur liée au cancer : stratégie et points forts du programme EFFADOL « Ensemble, Faire Face A la DOuLeur »

Therapeutic education and cancer pain: strategy and strengths of the EFFADOL program

Virginie Prevost ${ }^{1,2}$, Alexandra Leconte ${ }^{2}$, Natacha Heutte ${ }^{2}$, Claire Delorme ${ }^{3.4}$, Bénédicte Clarisse $^{2}$

1. UMR 1086 INSERM, Unité de Recherche Interdisciplinaire pour la Prévention et le Traitement des Cancers «ANTICIPE » et Université de Caen Normandie, Caen, France

2. Centre Régional de Lutte Contre le Cancer François Baclesse, Caen, France

3. Centre Hospitalier, Bayeux, France

4. Réseau Régional Douleur en Basse-Normandie, Bayeux, France

Correspondance : Virginie Prevost, UMR 1086 INSERM Cancers et Préventions Centre Francois Baclesse, Av. du Général Harris, 14076 CAEN cedex 05 virginie.prevost@unicaen.fr 
La douleur, dont la prévalence est évaluée à $59 \%$ en oncologie [1], reste parfois encore insuffisamment soulagée et altère la qualité de vie des patients [2]. Sa prise en charge se heurte à différents obstacles parmi lesquels le manque de connaissance et les représentations du patient et/ou de son entourage sur la douleur et ses traitements [3]. Afin de les surmonter, la participation active des patients s'avère nécessaire. L'éducation thérapeutique du patient (ETP) constitue une approche stratégique pertinente pour répondre à cette problématique [4], en permettant au patient de développer les compétences pour optimiser de manière active la gestion de sa douleur.

Sur notre territoire (départements du Calvados, de la Manche et de l'Orne), la prise en charge des patients atteints de cancer repose notamment sur deux réseaux structurés : le Réseau Régional de Cancérologie OncoNormandie et le Réseau Régional Douleur en Basse-Normandie (RRDBN) permettant ainsi la proximité et l'accessibilité pour tous à l'offre de soins. Le RRDBN s'appuie sur dix structures hospitalières (centres de référence et/ou de prise en charge de la douleur) pour chacune desquelles un binôme médecin-infirmière référent est identifié, permettant une couverture géographique régionale.

Dans ce contexte, nous portons un large programme de recherche d'éducation thérapeutique en matière de douleur liée au cancer (protocole EFFADOL pour «Ensemble, Faire Face A la DOuLeur »), qui s'inscrit dans une politique régionale de santé. L'élaboration, le déploiement et l'évaluation de ce programme se structure en cinq étapes (figure 1):

- Formation à l'ETP de professionnels de santé (10 binômes médecin/infirmière)

- Identification des attentes éducatives des patients et des proches dans le domaine de la douleur liée au cancer

- Conception d'un programme d'ETP sur la douleur liée au cancer, autorisé par l'Agence Régionale de Santé (ARS) 
- Evaluation régionale de la faisabilité, la qualité et la transférabilité du programme d'ETP

- Evaluation du programme d'ETP par randomisation comparative interventionnelle

Notre population cible est constituée de patients dont le cancer a été diagnostiqué depuis plus d'un mois, présentant des douleurs de fond non stabilisées (liées à la maladie et/ou à ses traitements), traitées par antalgiques depuis plus d'un mois. Les patients peuvent, s'ils le désirent, solliciter un proche pour participer à l'ETP.

Nous présentons la stratégie et les points forts de notre démarche, dans laquelle la mise en œuvre du programme d'ETP (étape 3), au-delà des attentes réglementaires, est enrichie par des étapes de recherche (étapes 2, 4 et 5), lui conférant une dimension d'expérimentation prospective.

Ainsi, en amont du programme, l'importance a été accordée à l'identification des besoins des patients en matière de douleur liée au cancer (étape 2), permettant de construire un programme pertinent répondant au mieux à leurs attentes. Cette identification a reposé sur un questionnaire administré à 75 patients dans le cadre d'une étude prospective multicentrique (référence ClinicalTrial NCT02391740), menée avec le soutien financier de la société TAKEDA.

En aval du programme, nous avons opté pour une évaluation en termes de qualité et d'efficacité à travers une étude régionale (étape 4) mais aussi à l'échelle nationale (étape 5). Cette dernière étape vise à évaluer le bénéfice de l'approche éducative par comparaison à la prise en charge conventionnelle de la douleur, en utilisant une méthodologie particulière, dite Step-Wedge [57]. Cette méthodologie répond à la nécessité d'éviter le risque de contamination entre les deux groupes et de réduire le risque de biais, la mise en place simultanée par les mêmes soignants de deux modes distincts de prise en charge de la douleur (ETP et prise en charge conventionnelle) n'étant pas envisageable pour évaluer une pratique impliquant une modification de posture du 
soignant. Elle permet d'échelonner la formation des soignants et par conséquent celle de l'intervention éducative à l'échelle des structures, la randomisation s'effectuant selon les centres (par grappe ou « cluster ») et non selon les patients.

Le critère de jugement retenu pour évaluer l'efficacité de la démarche d'ETP est la diminution du retentissement de la douleur sur les activités quotidiennes, mesurée à l'aide du Questionnaire Concis sur les Douleurs [8,9]. Nous avons retenu ce paramètre qui nous semble être plus pertinent et mieux refléter le bénéfice pour le patient en termes d'objectif éducatif que l'intensité de la douleur.

Les étapes d'évaluation du programme d'ETP en matière de douleur liée en cancer sont initiées, avec le soutien de la Ligue pour le Cancer et de l'Institut National du Cancer. Elles reposent sur une étude (référence ClinicalTrial NCT03297723) menée auprès de patients bénéficiant de l'ETP dans les centres du territoire bas-normand puis progressivement étendue à cinq structures nationales où seront inclus des patients avant puis après la formation des soignants à l'ETP, conformément à la méthodologie Step-Wedge pour comparer l'approche conventionnelle (avant la formation) à l'approche éducative.

L'originalité et la force de ce projet reposent sur un travail collaboratif entre acteurs de santé et chercheurs, s'appuyant sur des méthodologies robustes visant à objectiver les bénéfices apportés au patient de la démarche éducative.

Les principaux résultats attendus sont donc de démontrer l'efficacité de l'ETP pour améliorer les connaissances et les compétences des patients dans le domaine de la douleur afin de favoriser leur adhésion aux traitements et ainsi de s'impliquer pour mieux la gérer. L'objectif à long terme est de diffuser la démarche éducative se traduisant par une modification des pratiques apportant un bénéfice réciproque pour les patients et les soignants. 


\section{Remerciements}

Les auteurs remercient pour leur soutien le Pr Khaled Meflah (Directeur du Centre F. Baclesse), l'Espace Régional d'Education Thérapeutique (ERET) Basse-Normandie et le Dr LoudiyiMehdaoui (ARS). Les patients et représentants d'associations qui ont contribué à la construction de ce projet ainsi que l'ensemble des patients et des membres de leur entourage pour leur participation aux essais cliniques mis en œuvre dans le cadre de ce large programme de recherche sont également remerciés. Les auteurs remercient enfin pour leur soutien financier la Fondation Apicil et l'Association ARDCOM (étape 1), la société TAKEDA (étape 2), le Comité départemental de la Ligue contre le Cancer de Basse-Normandie (étape 4) et l'INCa (Appel d'offre RISP15-007_FP, étape 5).

Déclaration de liens d'intérêts : Les auteurs déclarent ne pas avoir de liens d'intérêts. 


\section{REFERENCES}

[1] Van den Beuken-van Everdingen MHJ, Hochstenbach LMJ, Joosten EAJ, Tjan-Heijnen VCG, Janssen DJA. Update on Prevalence of Pain in Patients With Cancer: Systematic Review and Meta-Analysis. J Pain Symptom Manage 2016;51:1070-90.

[2] Kroenke K, Theobald D, Wu J, Loza JK, Carpenter JS, Tu W. The association of depression and pain with health-related quality of life, disability, and health care use in cancer patients. J Pain Symptom Manage 2010;40:327-41.

[3] Brant JM. The global experience of cancer pain. Asian Pac J Cancer Prev 2010;11 Suppl $1: 7-12$.

[4] Oldenmenger WH, Gearing JI, Mostovaya I, Vissers KCP, de Graeff A, Reyners AKL et al. A systematic review of the effectiveness of patient-based educational interventions to improve cancer-related pain. Cancer Treat Rev 2018;63:96-103.

[5] Rasmussen CD, Hotelman A, Mortensen OS, Sugared K, Jorgensen MB. Prevention of low back pain and its consequences among nurses' aides in elderly care: a steppedwedge multi-faceted cluster-randomized controlled trial. BMC Public Health 2013;13:1088.

[6] Hussey MA, Hughes JP. Cluster randomized crossover trials: design and analysis of the stepped wedge design. Contemp Clin Trials 2007;28:182-91.

[7] Mdege ND, Man MS, Taylor CA, Torgerson DJ. Systematic review of stepped wedge cluster randomized trials shows that design is particularly used to evaluate interventions during routine implementation.J Clin Epidemiol 2011;64:936-48.

[8] Cleeland CS \& Ryan KM. Pain assessment : global use of the Brief Pain Inventory, Ann Acad Med Singapore 1994;13:129-38.

[9] Larue F, Colleau SM, Brasseur L, Cleeland CS. Multicentre study of cancer pain and its treatment in France. BMJ 1995;310:1034-7. 
Figure 1. Stratégie du programme EFFADOL «Ensemble, Faire Face A la DOuLeur » en cinq étapes

\section{CONTEXTE:}

Améliorer la gestion de la douleur liée au cancer : un défi

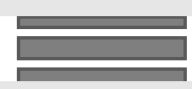

\section{ETAPE 1}

Formation des soignants à l'ETP'

10 binômes médecin-infirmière

\section{ETAPE 2}

Identification des attentes et des besoins des patients

Etude prospective multicentrique (75 patients)

\section{Caractérisation de la} population cible Evaluation des outils

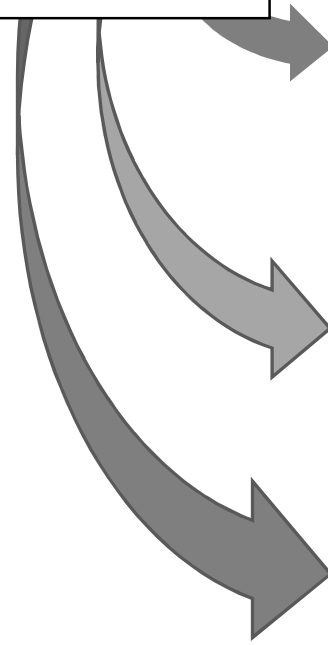

\section{ETAPE 3}

Développement, mise en œuvre et optimisation du programme (Validé par l'ARS')

\section{ETAPE 4}

Etude régionale

(Evaluation de la qualité et de la transférabilité du programme (90 patients)

\section{ETAPE 5}

Evaluation de l'efficacité du programme Par randomisation comparative interventionnelle, selon le design Step-Wedge

260 patients répartis comme suit :

-90 patients sur le territoire bas-normand (communs à l'étape 4) -Pour chacun des 5 centres hors du territoire bas-normand:

17 patients avant la formation des soignants à l'ETP

17 patients après la formation à l'ETP
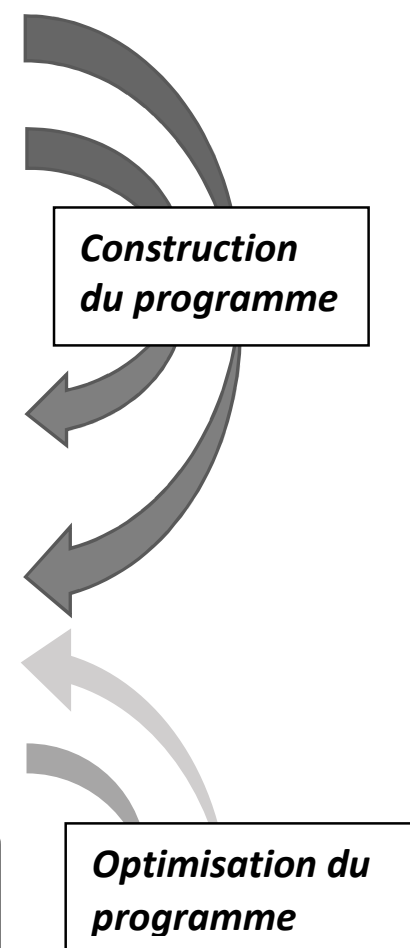

\section{RESULTATS ATTENDUS:}

Améliorer les compétences des patients leur permettant de mieux gérer leur douleur au quotidien Démontrer l'efficacité du programme et diffuser l'approche éducative

${ }^{1}$ Education Thérapeutique du Patient

${ }^{2}$ Agence Régionale de Santé 